\title{
Discontinued Splenogonadal Fusion and Bilateral Empty Scrotum in an 18-Month-Old Boy
}

\author{
Ahmed Abokrecha ${ }^{1}$ Ameera Almatrfi ${ }^{2}$ \\ ${ }^{1}$ Department of Pediatric Surgery, Maternity and Children Hospital, \\ Makkah, Saudi Arabia \\ 2 Department of Medicine and Surgery, Umm Al-Qura University \\ Faculty of Medicine, Makkah, Saudi Arabia \\ Eur J Pediatr Surg Rep 2017;5:e1-e3.
}

Address for correspondence Ameera Almatrfi, Department of Medicine and Surgery, Umm Al-Qura University Faculty of Medicine, Makkah 21955, Saudi Arabia (e-mail: ameera.almatrfi@gmail.com).

\begin{abstract}
Keywords

- Bilateral Empty Scrotum

- orchidopexy

- discontinued splenogonadal fusion

Splenogonadal fusion is a rare benign congenital anomaly defined as the presence of splenic tissue adherent to gonads. It was first described in 1883 by Bostroem, a German pathologist. We present a case of an 18-month-old boy who was referred as a case of bilateral empty scrotum since birth. During routine laparoscopic exploration, right vas deferens and testicular vessels were entering the right internal inguinal ring so right inguinal exploration was done, which revealed blind ending vas deferens and testicular vessels and the left testis was found intra-abdominally near the left internal ring with a mass on its upper pole. Wedge biopsy was taken from the upper pole of the testicle (site of the mass) for tissue diagnosis followed by orchidopexy. Histology showed splenic tissue. Although splenogonadal fusion is a rare condition, surgeons should be aware of this rare disease entity to avoid unnecessary aggressive interventions such as orchiectomy.
\end{abstract}

\section{Introduction}

Splenogonadal fusion is a rare benign congenital anomaly defined as the presence of splenic tissue adherent to gonads. It was first described in 1883 by Bostroem, a German pathologist. This condition is usually discovered incidentally during inguinal hernia or a testicular mass. ${ }^{1-3}$ There are approximately 175 cases reported in the literature that showed left side and male predominance. Splenogonadal fusion can be subgrouped into continuous and discontinuous types based on splenic attachments. ${ }^{4}$ It commonly presents as inguinal hernia or testicular mass and may be misdiagnosed as testicular malignancy; in some cases of continuous types, it might be associated with congenital anomalies such as limb defects, microganthia, cleft palate, and cardiac defects. ${ }^{3,5}$ We present the case of an 18-month-old boy who was referred as a case of bilateral empty scrotum since birth.

\section{Case Report}

An 18-month-old boy was referred as a case of bilateral empty scrotum since birth. When we reviewed the case, we found a male baby of average weight and height with uncircumcised well-formed penis and urethral meatus in normal position with bilateral impalpable undescended testes. There were no associated anomalies. The patient was scheduled for laparoscopic exploration and orchidopexy, if possible. Laparoscopic exploration revealed no abnormalities and the spleen in its normal place. On the right side, the vas deferens and testicular vessels were attenuated, atrophic, and entering the right deep inguinal ring. Therefore, an exploration of the right inguinal region was performed, which revealed a blind ending vas deferens and testicular vessels. The left testicle was found intra-abdominally near the left internal ring and therefore the testicle was delivered easily to the left inguinal region through an inguinal incision with minimal dissection. We received

August 16, 2016

accepted after revision

January 5, 2017
DOI http://dx.doi.org/

$10.1055 / \mathrm{s}-0037-1598624$.

ISSN 2194-7619.
(C) 2017 Georg Thieme Verlag KG Stuttgart · New York
License terms

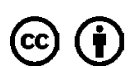




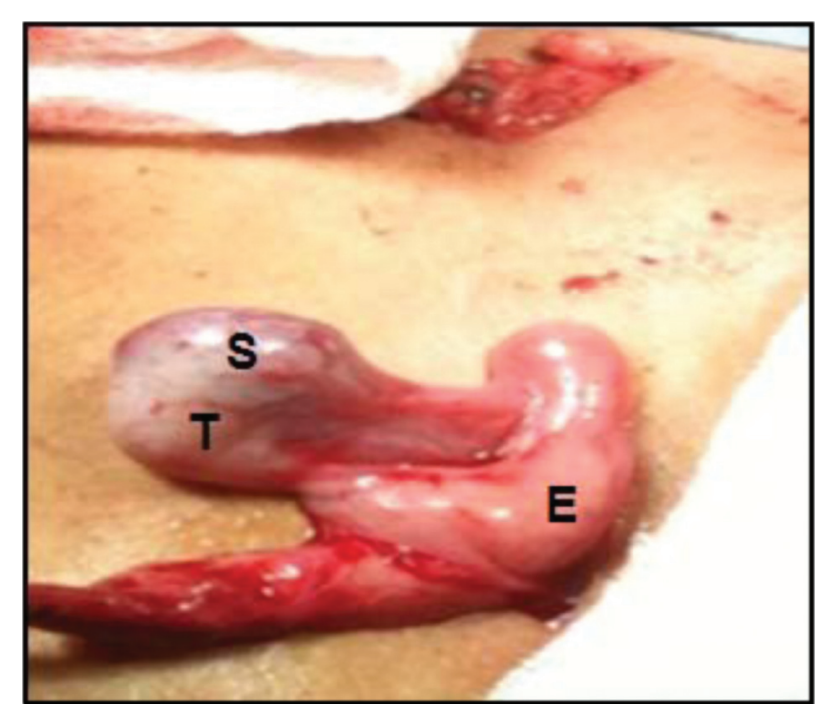

Fig. 1 Figure shows the left testicle. S, Splenic tissue; T, testis; E, epididym.

noticed a mass of the upper pole of the left testicle with no clear demarcation between the mass and the testicular parenchyma. The surface of the mass was smooth, irregular in shape, and firm ( - Fig. 1). The options were either of the following: (1) orchiectomy including the mass if considered as malignant, but the patient had no other testicle, (2) excisional biopsy of the mass and orchidopexy, which was not feasible, or, lastly, (3) incisional biopsy of the mass and scrotal orchidopexy. A wedge biopsy was taken from the upper pole of the testicle (site of the mass) for tissue diagnosis followed by orchidopexy. The patient passed uneventful postoperative days. Histopathology showed no malignant or inflammatory cells but lymphatic (splenic) tissue. The diagnosis of splenogonadal fusion (discontinuous type) of intra-abdominal left undescended testis and vanished right testicle was made. As the patient had only one testicle with a splenic tissue remnant in the upper pole, the plan for meticulous dissection to excise the residual splenic tissue was made. The child was booked for complete excision of mass from the upper pole of left testicle after 6 months of observation.

\section{Discussion}

Splenogonadal fusion is a rare benign congenital anomaly defined as the presence of splenic tissue adherent to the gonads discovered incidentally. ${ }^{1-3,6}$ Male-to-female ratio is approximately 16:1 with predominance on the left side. Splenogonadal fusion can be subgrouped based on the attachment to the spleen into a continuous and discontinuous type. $^{4,7}$ The continuous type represents approximately $55 \%$ of cases and is characterized by a connection between the spleen and the gonads through a cord of splenic or fibrous tissue. The discontinuous type (44\% of cases) has no direct connection of the gonads with accessory spleens or ectopic splenic tissue., ${ }^{3,7}$ The exact pathogenesis for splenogonadal fusion is not yet understood, but it is thought to develop between the fifth and the eighth week of gestation based on the close location between the splenic tissue and left gonadal ridge. When the spleen develops in the dorsum of the mesogastrium from a group of mesenchymal cells, the gonads start to develop in the posterolateral wall of the embryo at the same time. During embryonic gut rotation, the spleen development comes into close approximation to the left urogenital fold. Furthermore, subsequent inflammation or adhesions on the peritoneal surface may facilitate splenogonadal fusion. ${ }^{4,7-9}$ This condition commonly presents as an inguinal hernia or a testicular mass and may be misdiagnosed with testicular malignancy. In the literature, four cases of malignancies associated with splenogonadal fusion have been described. These patients presented as adults with different histopathological findings and, in cases of the continuous type, with congenital anomalies such as limb defects, microganthia, cleft palate, and cardiac defects. ${ }^{3,5,10}$ The diagnosis is usually made by ultrasonography. In case of insufficient resolution, a computed tomography or magnetic resonance imaging, and lastly technetium-99m spleen scintigraphy, which is the most recommended tool in the literature, may be performed. ${ }^{3,7}$ However, the most useful diagnostic test is surgical exploration. In our case, the availability of frozen section in first operation saved the child from orchiectomy, but frozen section may not be available at all times. ${ }^{3,4,11,12}$ Splenogonadal fusion is a benign condition. Surgery is not required if clinical signs are absent, especially in the scrotum. If surgery is performed, the testis can be preserved as the splenic tissue can usually be easily separated from the gonad. ${ }^{13}$

\section{Conclusion}

Splenogonadal fusion is a rare benign congenital anomaly. Pediatric surgeons should be aware of this disease entity to avoid unnecessary aggressive interventions such as orchiectomy.

\section{References}

1 Shadpour P, Rezaimehr B. "Rosary of Testes": splenogonadal fusion in association with bilateral abdominal testes presenting as polyorchidism. Case Rep Urol 2015;2015:317189

2 Celik A, Tiryaki S, Darcan S, Ergun O. Splenogonadal fusion-limb deformity syndrome: a rare but important cause of undescended testis. World J Pediatr 2016;12(02):246-248

3 Bosnalı O, Cici İ, Moralıoğlu S, Cerrah-Celayir A. Continuous-type splenogonadal fusion: report of a rare case. Turk J Pediatr 2014; 56(06):680-683

4 Melikov R, Altunkol A, Quliyev F, Mammadov E, Abat D. Continuous-type splenogonadal fusion: a rare cause of scrotal swelling. Pediatr Urol Case Rep 2015;2(02):22

5 Lakshmanan PM, Reddy AK, Nutakki A. A surprising content of congenital hernia: complete splenogonadal fusion band. BMJ Case Rep 2014;2014(14:pii:bcr2014203640

6 Kennedy KP, Barnard S, Speakman MJ. Splenogonadal fusion - not just another hydrocoele. Ann R Coll Surg Engl 2006;88(02):163-164

7 Ferrón SA, Arce JD. Discontinuous splenogonadal fusion: new sonographic findings. Pediatr Radiol 2013;43(12):1652-1655 
8 Milliken I, Cherian A, Najmaldin A, Powis MR. Splenogonadal fusion: a rare cause of testicular enlargement. Pediatr Surg Int 2007;23(04):365-367

9 Chen SL, Kao YL, Sun HS, Lin WL. Splenogonadal fusion. J Formos Med Assoc 2008;107(11):892-895

10 Imperial SL, Sidhu JS. Nonseminomatous germ cell tumor arising in splenogonadal fusion. Arch Pathol Lab Med 2002;126(10): 1222-1225
11 Hasegawa Y, Kanemitsu I,Ueoka K. Transverse testicular ectopia with splenogonadal fusion: a case report. Int J Urol 2011;18(06):466-468

12 Zhang X-w, Hou G-j, Geng X-j, Gao X-y, Chen Y-j, Fu L-b. Splenogonadal fusion: Report of two cases and literature review. J Pediatr Surg Case Rep 2013;1(10):362-363

13 Li WF, Luan MX, Ma Z, Chen YJ. Splenogonadal fusion: report of four cases and review of the literature. Exp Ther Med 2013;6(03): 816-818 